\title{
Morphons: Segmentation using elastic canvas and paint on priors
}

\author{
Hans Knutsson and Mats Andersson
}

\section{Linköping University Post Print}

\section{Tweet}

N.B.: When citing this work, cite the original article.

Original Publication:

Hans Knutsson and Mats Andersson, Morphons: Segmentation using elastic canvas and paint on priors, 2005, Image Processing, 2005. ICIP 2005. IEEE International Conference. Vol. 2, 1226-1229.

http://dx.doi.org/10.1109/ICIP.2005.1530283

(C2005 IEEE. Personal use of this material is permitted. However, permission to reprint/republish this material for advertising or promotional purposes or for creating new collective works for resale or redistribution to servers or lists, or to reuse any copyrighted component of this work in other works must be obtained from the IEEE.

\section{http://ieeexplore.ieee.org/}

Postprint available at: Linköping University Electronic Press http://urn.kb.se/resolve?urn=urn:nbn:se:liu:diva-28792 


\title{
MOR P H ONS : SEGMENTATION USING ELASTIC CANVAS AND PAINT ON PRIORS
}

\author{
Hans Knutsson Mats Andersson \\ Medical Informatics, Dept. of Biomedical Engineering \& \\ Center for Medical Image Science and and Visualization \\ Linköping University, Linköping Sweden
}

\begin{abstract}
This paper presents a new robust approach for segmentation. The segmentation is attained by morphing of an $N$ dimensional model, the Morphon, onto the $N$-dimensional data. The approach is general and can, in fact, be said to encompass much of the deformable model ideas that have evolved over the years. However, in contrast to commonly used models, a distinguishing feature of the Morphon approach is that it allows an intuitive interface for specifying prior information, hence the expression paint on priors. In this way it is simple to design Morphons for specific situations.

The priors determine the behavior of the Morphon and can be seen as local data interpreters and response generators. There are three different kinds of priors: - material parameter fields (elasticity, viscosity, anisotropy etc.), - context fields (brightness, hue, scale, phase, anisotropy, certainty etc.) and - global programs (filter banks, estimation procedures, adaptive mechanisms etc.). The morphing is performed using a dense displacement field. Both the material parameter and context fields are addressed via the present displacement field. An example of the performance of is given using 2D ultrasound images of a heart where the purpose is to segment the heart wall.
\end{abstract}

\section{INTRODUCTION}

The history of image segmentation goes back to the very beginning of image processing and is still a topic of major concern. Naive thresholding based approaches are basically abandoned and the need to incorporate strong prior information in non-trivial segmentation tasks is disputed by no one. A priori information in terms of allowable deformation of a prototype object has been frequently used in segmentation of 2D images and for tracking of deformable objects in video. Examples of such algorithms are deformable templates/prototypes [1, 2], trainable snakes [3], balloon models[4] and more recent approaches [5, 6, 7].

To fit a prototype to an image these methods generally use image related and geometric terms. The image features are typically based on edge detection for alignment of the object boundary. The geometric deformation of the prototype can e.g. be controlled by limiting the deformation of the object to a low order parametric motion model or introduce a triangular meshgrid and define the stiffness of the prototype using a FEM model.

Existing deformable models are however sensitive to the initial conditions of the computation and often get trapped in local minima. In such cases interactive measures are paramount to attain successful results. For most users interacting with the deformable model is complicated and non intuitive. In addition there are typically no means for user interaction during the extraction process. This paper presents a new method for segmentation which is both robust and has intuitive parameters settings.
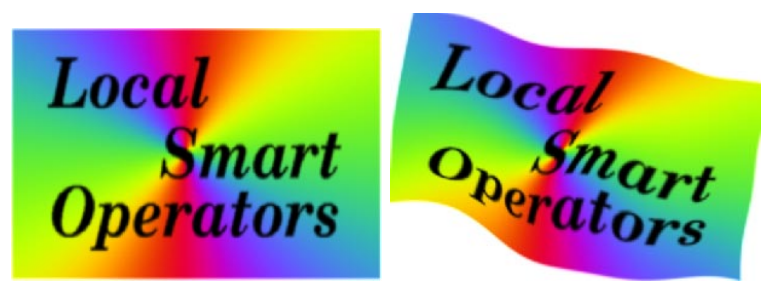

Fig. 1. The Morphon acts like an elastic canvas with painted on smart local operators each determining where to go next.

Figure 1 is meant to illustrate the basic mode of operation of the Morphon. The priors form a field with different individual priors at each location of an elastic canvas (of $N$ dimensions). The priors determine how the neighborhood will be perceived and how this percept translates into an appropriate new location. Thus the canvas is morphed according to the outputs of the 'smart operators' that are 'painted' on the canvas.

\section{MORE ON MORPHONS}

Many different types of priors can be attached to the morphon and it is helpful to group the priors into three groups: material, context and program. 


\section{- Material}

Parameter field determining the local 'material' properties. The fact that the effect of these parameters are mediated through spatio-temporal filtering is a novel feature of the Morphon approach. The term 'material' need not be given a strict physical interpretation but gives intuitively useful associations. Typical properties that are locally determined are elasticity, viscosity, anisotropy and memory behavior.

\section{- Context}

Parameter field holding information that support interpretation of the image data. Typically one part of the context is of the same type as the data at hand, e.g. intensity. Other types of information that can be present in the context is, for example, local scale, orientation, phase, anisotropy and certainty.

\section{- Program}

Global definition of what to do, how to use priors and data and what outputs to produce. Typical examples are filter banks, estimation algorithms and adaptive mechanisms.
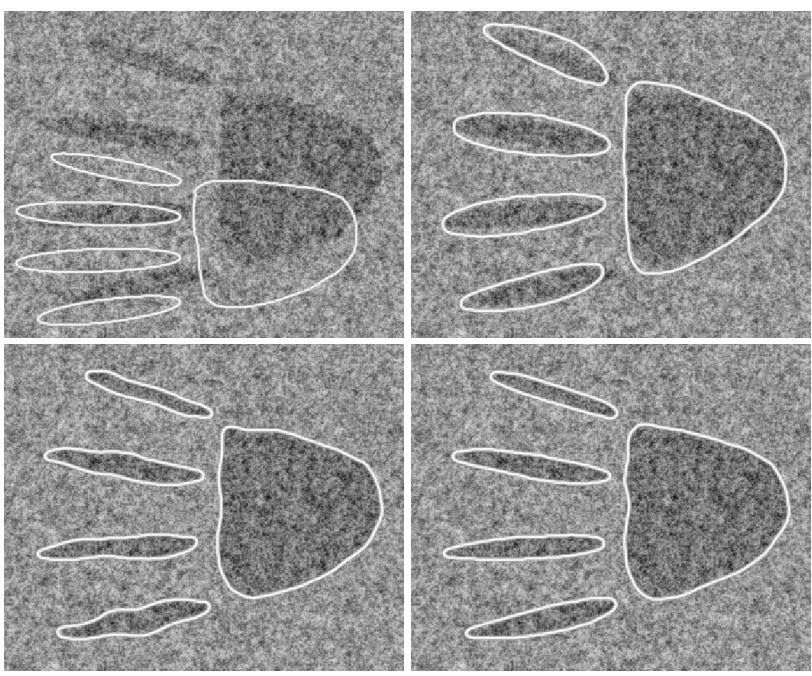

Fig. 2. Top left: Initial position of the 'hand' Morphon (white sketch) and image. Top right: Result using 'stiff', material. Lower left: 'Medium stiff' material. Lower right: Anisotropic material.

Figure 2 shows the initial situation and three different segmentations of a stylized 'hand' object. The main mode of freedom of the hand is that the fingers can be spread. The initial Morphon hand has the fingers much closer together than the hand in the image. All three segmentation results are in some sense successful in that all fingers are found. However, fig. 2 shows how the segmentation result can be significantly improved by using material parameters that reflect the properties of the object.

If a stiff material is used the Morphon fingers still can be spread apart but they will get thicker as the material will resist non-linear displacement attempts. If the stiffness is reduced the fingers are allowed to retain their original thickness but the noise in the image now introduces wiggles (which is not a natural mode of variation of fingers). The solution is to give the material anisotropic stiffness, i.e. a material that is 'soft' in the finger spreading direction but resists variations in displacement along the fingers, see the lower right result in fig. 2. This illustrates the straightforward relation between the modes of variation of the object class to be segmented and the 'painting' of the priors.

\section{HEART WALL SEGMENTATION FROM 2D ULTRASOUND IMAGES.}
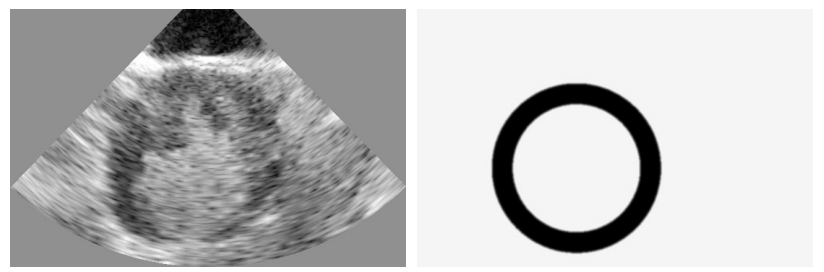

Fig. 3. Left: The original ultrasound image of a heart. Right: The Morphon context field.

As an initial evaluation of the performance, the Morphon approach is used to segment out the heart wall in an ultrasound image of a heart. This is a very difficult task even for trained medicals. The ultrasound image is shown in the left part of fig. 3. The presence of contrast agent in the blood makes the heart wall appear darker than the surrounding tissue and the blood pool. The dark objects in the interior of the heart is tissue not belonging to the heart wall. The grey level in the right part of fig. 3 defines the context field for the initial state of the Morphon. The heart wall is expected to be a closed object with an approximate size and wall thickness. Note that this context field provides itself to a very simple interface to a human operator.

\subsection{Morphing the Morphon}

The adaptation of the Morphon is an iterative process using a multitude of scales. A central part of the algorithm is to compute a dense displacement field that morphs the initial context fields of the Morphon onto the target, in this case the heart wall. The computation of the displacement field is not critical since the algorithm is iterative. There exists a large variety of displacement/motion estimation algorithms, pick your favorite. The authors prefer a phase-based quadrature filter approach which is briefly described in section 3.4. 

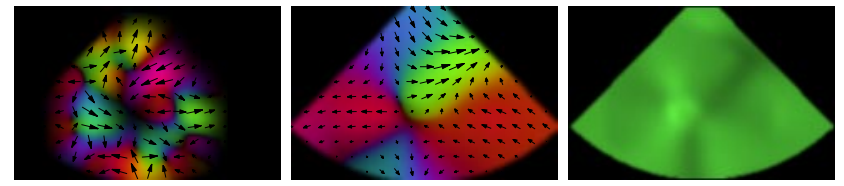

Fig. 4. Left: Incremental displacement field for scale $i$, $\boldsymbol{d}_{i}$. Center: Accumulated displacement field, $\boldsymbol{d}_{a}$. The mean value is subtracted. Right:The determinant of the Jacobian of $\boldsymbol{d}_{a}$.

A dense displacement field is accumulated from a number of iterations from coarse to fine scale. At each scale the present accumulated displacement field, $\boldsymbol{d}_{a}$, is updated by an additional displacement field for the current scale, $\boldsymbol{d}_{i}$. Performing 2-5 iterations on each scale makes the precision of the of incremental displacement field less critical. In fig. 4 the accumulated and incremental displacement fields are illustrated at an intermediary scale of the adaptation process. The method by which these fields and their associated certainties are combined and updated comprise a central part of the proposed algorithm.

\subsection{Displacement field accumulation}

For the moment we leave the details of the displacement and certainty computation and focus on the accumulation of the displacement fields with respect to the estimated certainties. For each iteration the following fields are computed.

Accumulated displacement and certainty: $\boldsymbol{d}_{a} c_{a}$

Incremental displacement and certainty: $\boldsymbol{d}_{i} \quad c_{i}$

Since the certainty field for iteration $i$ comprise a total displacement corresponding to $\boldsymbol{d}_{a}+\boldsymbol{d}_{i}$ it is natural to update the accumulated displacement field as:

$$
\boldsymbol{d}_{a}=\frac{c_{a} \boldsymbol{d}_{a}+c_{i}\left(\boldsymbol{d}_{a}+\boldsymbol{d}_{i}\right)}{c_{a}+c_{i}}
$$
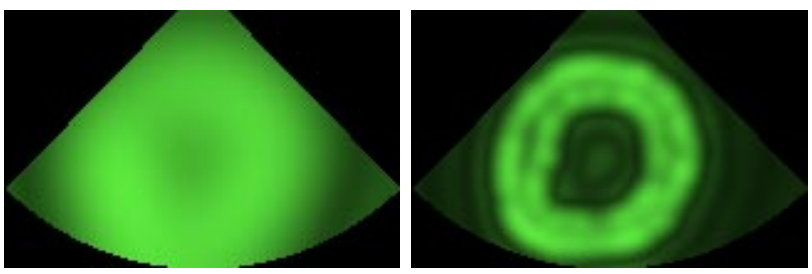

Fig. 5. Left: certainty in an early stage of the morphing process. Right: certainty in the final state.

From eq. (1) it is clear that the impact of $\boldsymbol{d}_{i}$ is very small unless the certainty for the current iteration, $c_{i}$, is significant compared to the accumulated certainty, $c_{a}$.

The adaptation of the accumulated certainty, $c_{a}$, is a bit more complicated as it would be desirable to have certainty estimates of the certainty estimates themselves. A straightforward method to solve this problem is simply to use $c_{a}$ and $c_{i}$ as their own certainties, i.e. update certainties as

$$
c_{a}=\frac{c_{a}^{2}+c_{i}^{2}}{c_{a}+c_{i}}
$$

In practice it turns out that it is beneficial to increase the weight $c_{i}$ for a fine resolution scale to maintain full adaptivity of the Morphon over all scales. Introducing the scale parameter, $\rho$, the accumulated certainty is computed as

$$
c_{a}=\frac{c_{a}^{2}+\left(2^{-\rho} c_{i}\right)^{2}}{c_{a}+2^{-\rho} c_{i}}
$$

where $\rho=0$ at original resolution and $\rho>0$ at the coarser scales. In this example the scales are separated by half an octave comprising a total of 3 octaves in scale space. Figure 5 shows how the accumulated certainty evolves during the adaptation from coarse to fine scale.

\subsection{Normalized regularization of the displacement field}

In the general case there exists a large class of displacement fields that result in practically identical Morphon context fields. In most cases we prefer the most simple displacement field. This provides a more robust algorithm. For this reason the incremental displacement field is subject to a regularization at each iteration. The regularization is performed by filtering using a Gaussian lowpass kernel, $g$, and normalized convolution with respect to $c_{i}$.

$$
\boldsymbol{d}_{i}=\frac{\left(c_{i} \boldsymbol{d}_{i}\right) * g}{c_{i} * g}
$$

The overall 'stiffness' of the morphon is defined by size of the Gaussian filter and the variance of $g$ is decreased for finer scales. In this example the context field of the Morphon has isotropic stiffness.

To monitor the regularization of the displacement field the determinant of the Jacobian of the displacement field is computed describing the local area change of the Morphon context field. Negative values in the determinant field implies that the prototype is 'folded over'. If this is the case a more powerful regularization is applied. The right part of fig. 4 shows the determinant of the Jacobian of the accumulated displacement field.

\subsection{Phase based estimation of the displacement field}

There exists a large variety of displacement/motion estimation algorithms that can be used for the computation of the displacement field. There is however one common issue that must be considered. Due to the aperture problem the displacement field can not be unambiguously estimated for all neighbourhoods. In these cases it is important to maintain 
as large degree of freedom as possible and not unnecessarily prevent displacements along the border of the Morphon.

In the present example the displacement estimate is, for each scale, based on conjugate products of quadrature filter [8] responses, i.e. $\boldsymbol{Q}=\boldsymbol{q}_{M} \boldsymbol{q}_{T}^{*}$ where $\boldsymbol{q}_{M}$ and $\boldsymbol{q}_{T}$ are the responses for the present state of morphon context field and the target image respectively. These estimates are performed in four directions and the final displacement estimate, $\boldsymbol{v}$, is obtained as the solution of a least squares problem:

$$
\min _{\boldsymbol{v}} \sum_{k=1}^{4}\left[c_{k} \hat{\boldsymbol{n}}_{k}^{T} \boldsymbol{T}\left(\hat{\boldsymbol{n}}_{k} d_{k}-\boldsymbol{v}\right)\right]^{2}
$$

Where:

$$
\begin{array}{lr}
c_{k}=\sqrt{\left|\boldsymbol{Q}_{k}\right|}\left(1+\Re\left(\hat{\boldsymbol{Q}}_{k}\right)\right) & \text { certainty in direction } k \\
\hat{\boldsymbol{n}}_{k} & \text { direction of filter } k \\
\boldsymbol{T} & \text { local structure tensor, see }[9,10] \\
d_{k} \propto \arg \left(\boldsymbol{Q}_{k}\right) & \text { displacement estimate in direction } k . \\
\boldsymbol{v}=\left(v_{x}, v_{y}\right)^{T} & \text { estimated displacement }
\end{array}
$$

\subsection{Final result}

Figure 6 shows the initial and final position of the Morphon context field. The proposed method shows robust behavior using the noisy ultrasound image sequence and the simple priors. The algorithm provides a simple and intuitive interface for an operator that is not specialized in image processing.

\section{ACKNOWLEDGMENT}

This work was funded by the Swedish Agency for Innovation Systems (VINNOVA) and Amersham Health within the NIMED program which is greatfully acknowledged. We are grateful to Birgitta Janerot Sjöberg, MD, PhD, for providing the ultrasound data sequence and valuable clinical support.

\section{REFERENCES}

[1] Anil K. Jain, Yu Zhong, and Sridhar Lakshmanan, "Object matching using deformable templates," IEEE Trans. Pattern Anal. Mach. Intell., vol. 18, no. 3, pp. 267-278, 1996.

[2] G. Klein, Deformable models for volume feature tracking, Ph.D. thesis, University of California, Berkeley, 1999.

[3] A. Baumberg and J. Hogg, "Generating spatiotemporal models from examples," Image and Vision comput., vol. 14, no. 8, pp. 525-532, 1996.
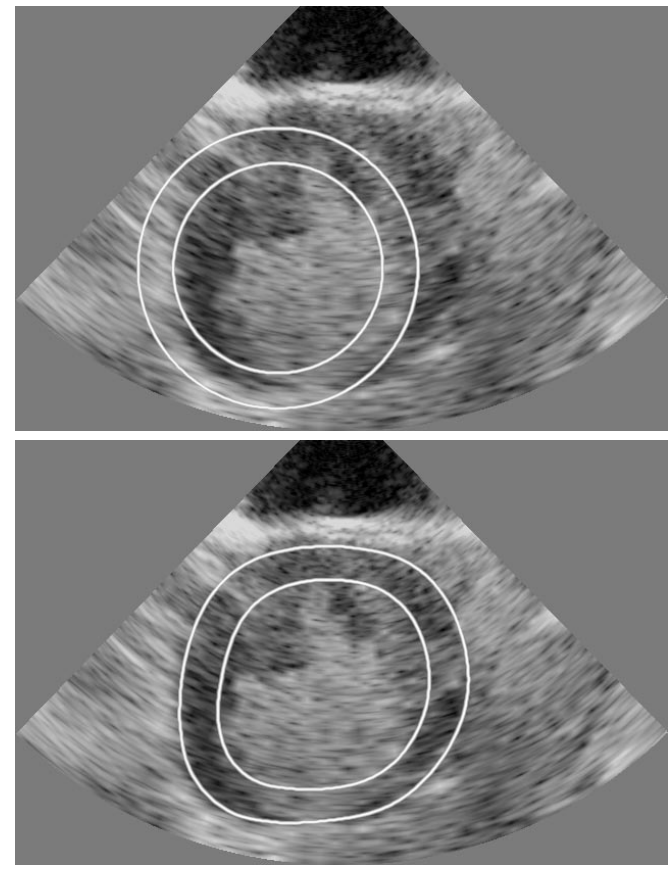

Fig. 6. Top: initial position of Morphon Bottom: final adaptation of prototype pattern.

[4] L. D. Cohen, "On active contour models and balloons," Computer Vision, Graphics, and Image Processing. Image Understanding, vol. 53, no. 2, pp. 211218, 1991.

[5] Stan Sclaroff and John Isidoro, "Active blobs: regionbased, deformable appearance models," Comput. Vis. Image Underst., vol. 89, no. 2/3, pp. 197-225, 2003.

[6] D. Cremers and C. Schnörr, "Motion competition: Variational integration of motion segmentation and shape regularization," German Conf. on Pattern Recognition (DAGM), 2002.

[7] D. Cremers, "Bayesian approaches to motion-based image and video segmentation," 1st International Workshop on Complex Motion, DAGM, 2004.

[8] H. Knutsson, Filtering and Reconstruction in Image Processing, Ph.D. thesis, Linköping University, Sweden, 1982, Diss. No. 88.

[9] H. Knutsson, "Representing local structure using tensors," in The 6th Scandinavian Conference on Image Analysis, Oulu, Finland, June 1989, pp. 244-251, Report LiTH-ISY-I-1019, Computer Vision Laboratory, Linköping University, Sweden, 1989.

[10] G. H. Granlund and H. Knutsson, Signal Processing for Computer Vision, Kluwer Academic Publishers, 1995, ISBN 0-7923-9530-1. 